\title{
Comparison of unscheduled re-attendance and contraception at discharge, among women having the final stage of early medical abortion at home and those remaining in hospital
}

\author{
Hannah Astle, ${ }^{1}$ Sharon T Cameron, ${ }^{2}$ Anne Johnstone ${ }^{3}$
}

\begin{abstract}
'Medical Student, College of Medicine and Veterinary Medicine, University of Edinburgh, Edinburgh, UK ${ }^{2}$ Consultant Gynaecologist, Chalmers Sexual Health Centre, and Royal Infirmary of Edinburgh, Edinburgh, UK ${ }^{3}$ Clinical Research Nurse, Department of Reproductive and Developmental Sciences, University of Edinburgh, Edinburgh, UK
\end{abstract}

\section{Correspondence to}

Dr Sharon T Cameron, Chalmers Sexual Health Centre, 2a Chalmers Street, Edinburgh EH3 9ES, UK:

sharon.cameron@ed.ac.uk

Received 19 May 2011 Accepted 12 July 2011 Published Online First 13 September 2011

\begin{abstract}
Introduction Throughout Great Britain, increasing numbers of women having an early medical abortion are choosing to go home soon after administration of misoprostol, to expel the pregnancy at home (early medical discharge, EMD), rather than remain upon the hospital premises (day case). However, data are lacking on how this impacts upon an abortion service in terms of unscheduled re-attendance rates and contraception provision at discharge.

Methods A retrospective audit was carried out of women undergoing medical abortion (up to 64 days' gestation) over 9 months at a National Health Service hospital in Scotland, to determine (1) unscheduled re-attendance rates within 6 weeks of the procedure for an abortion-related complication and (2) method of contraception provided at discharge.

Results Over the audit period 1128 women had an early medical abortion of whom 590 (52\%) chose EMD. There was no significant difference in unscheduled re-attendance rates between $\operatorname{EMD}(n=23,4 \%)$ and day case groups $(n=20,4 \%)$. There was no significant difference in the proportion of women in each group who left hospital with an effective method of contraception ( $n=362,61 \%$ and $n=355,60 \%$ for EMD and day case groups, respectively).
\end{abstract}

Conclusions Women undergoing early medical abortion who choose to expel the pregnancy at home are no more likely to re-attend hospital with a post-abortal complication and are just as likely to receive effective contraception than those who remain on hospital premises.

\section{Introduction}

In most countries throughout the world where early medical abortion with mifepristone and misoprostol is available (including the USA, France and Sweden) women who are in early pregnancy and who request a medical method of abortion

\section{Key message points}

Women who choose to go home to pass the final stage of early medical abortion there do not have higher rates of unscheduled re-attendance than women treated as a day case.

- Permitting women to go home soon after misoprostol administration does not affect provision or uptake of effective methods of contraception.

are able to choose to administer misoprostol at home and to expel the pregnancy at home. ${ }^{1-4}$ Although there is a growing evidence base that 'home' medical abortion is both safe and acceptable to women, ${ }^{1-4}$ the interpretation of the Abortion Act 1967 is that (in Great Britain) misoprostol is required to be administered on licensed premises. $^{5}$ Increasing numbers of women at early gestation who request medical abortion in Great Britain are, however, choosing to go home soon after misoprostol administration, to pass the final stage of the procedure at home. ${ }^{6-8}$ This early medical abortion service that permits women to go home soon after administration of misoprostol has previously been termed 'early medical discharge' (EMD), to distinguish it from 'day case' medical abortion where women are admitted to a medical abortion unit to pass the pregnancy, usually for a period of up to 6 hours. ${ }^{6}$ Studies throughout Great Britain have demonstrated high levels of acceptability of EMD among women who choose this service. ${ }^{6-9}$ Data are, however, lacking on how an EMD service impacts upon a National Health Service (NHS) abortion service in Great Britain, and in particular whether or not women who choose EMD have differential unscheduled re-attendance rates at the service for 
a problem related to the procedure compared to counterparts treated as day cases. There are also concerns that since women choosing EMD do not remain on the abortion service premises, they may be less likely to be provided with an effective method of contraception at discharge.

In Scotland, the vast majority of all abortions are performed in the NHS and almost exclusively within a hospital setting. ${ }^{10}$ The Royal Infirmary of Edinburgh (RIE) is a major teaching hospital in Edinburgh and is the main provider of abortion services in Lothian (Edinburgh and the surrounding area). In March 2009, an EMD service was introduced for women requesting an abortion at up to 8 weeks' gestation, who wished to avail themselves of this treatment and who fulfilled previously published criteria. ${ }^{6}$ From April 2010 the gestation limit for EMD at the RIE was raised to 9 weeks' gestation. As part of this EMD service, women who choose EMD return to the clinic for the second part of the medical abortion treatment with $800 \mu \mathrm{g}$ misoprostol administered vaginally, 24-48 hours after receiving $200 \mathrm{mg}$ oral mifepristone. This medical abortion regimen is identical to that used for women undergoing treatment as a day case, with the exception that women treated as a day case (i.e. admitted to a ward for up to 6 hours after misoprostol administration) may be given a further $400 \mu \mathrm{g}$ dose of misoprostol if they fail to abort within 4 hours of admission. An additional dose of misoprostol is not supplied to women choosing EMD since this medication must be administered on licensed premises. ${ }^{5}$ The analgesic regimen used for EMD has previously been described in detail, ${ }^{6}$ but is essentially the same for both EMD and day case treatment, with women in both groups receiving empirical analgesia (1 g paracetamol orally and $400 \mathrm{mg}$ ibuprofen orally, unless contraindicated) when misoprostol is administered, and dihydrocodeine offered for subsequent breakthrough pain if required. Women in EMD are given a small supply of dihydrocodeine tablets to take home. ${ }^{6}$ Contraception is dispensed to women in both EMD and day case treatment groups at discharge and women are provided with written information on what to expect over the following days, together with 24-hour emergency contact numbers. A follow-up appointment with the abortion service is arranged for all women choosing EMD 2 weeks later to confirm complete abortion, but only for those day case patients who fail to expel the pregnancy during their admission.

The main aim of this study was to determine if unscheduled re-attendance rates for a complication related to early medical abortion (up to 9 weeks' gestation) differed between those women who opted to be treated as EMD and those treated as a day case. A secondary aim was to compare the proportions of women having EMD and day case medical abortion who left the service with an effective ongoing method of contraception.

\section{Methods}

A retrospective audit was conducted of those patients who had a medical abortion as either EMD or a day case between January and September 2010 (inclusive) at the RIE. Women were identified using the admission book for the medical abortion unit. Demographic details on women including age, gestational age, reproductive history and postcode were recorded. Using the patient's postcode it was possible to obtain a Carstairs deprivation category. ${ }^{11}$ Data on method of contraception provided to women at discharge was routinely recorded in the admission book. Contraceptive advice and supplies are offered to all women to commence following misoprostol treatment. This includes provision of longacting reversible contraceptive (LARC) methods such as the progestogen-only injectable and insertion of a progestogen-only implant. For women who wish to use an intrauterine method, a 'fast-track' appointment for insertion is made for women at the local family planning service in central Edinburgh (Chalmers Sexual Health Centre). The admission book of the medical abortion unit also provided details on women who chose to be 'fast-tracked' for this process.

Details of any hospital re-attendances (within NHS Lothian) within 6 weeks of the procedure were gathered using the regional hospital computerised patient management system database of patient records (TRAK). Case notes of women who re-attended were then reviewed to collect full details on the reason for attendance, diagnosis and treatment instituted.

\section{Statistical analysis}

Statistical analysis was performed on coded data using IBM Statistical Package for the Social Sciences (SPSS) software Version 17 (SPSS Inc., Chicago, IL, USA). Comparisons were carried out using independent samples $t$-test and Chi-square $\left(\chi^{2}\right)$ or Fishers test where appropriate. Statistical significance was defined as $p<0.05$.

\section{Ethical approval}

The ethics committee responsible for medical students at the University of Edinburgh reviewed the study proposal and concluded that regional ethical committee approval was not required. The quality improvement team for gynaecology and the clinical effectiveness team for reproductive health at the RIE approved the audit.

\section{Results}

\section{Demographics of women}

A total of 1128 women underwent a medical abortion ( $\leq 64$ days' gestation) at the RIE during the study period, of which 590 (52\%) chose EMD and 538 (48\%) had treatment as a day case. Women choosing EMD were slightly but statistically older than those having a day case procedure (mean age 26.6 and 24.5 years for EMD and day case, respectively; $p=0.011$ ), with a higher proportion at $<50$ days' gestation $[n=369$ 
Table 1 Demographics of women having medical abortion by either early medical discharge or day case procedure

\begin{tabular}{|c|c|c|c|}
\hline Demographic & $\operatorname{EMD}(n=590)$ & Day case $(n=538)$ & $p$ \\
\hline Age (years) [mean (SD)] & $26.5(6.4)$ & $24.5(5.7)$ & 0.011 \\
\hline \multicolumn{4}{|l|}{ Parity $[n(\%)]$} \\
\hline Nulliparous & $322 \quad(55)$ & $357 \quad(66)$ & \multirow[t]{2}{*}{0.003} \\
\hline Parous & $268 \quad(45)$ & (34) & \\
\hline \multicolumn{4}{|l|}{ Gestation (days) $[n(\%)]$} \\
\hline$<50$ & $369 \quad(62)$ & 231 & \multirow[t]{2}{*}{$<0.001$} \\
\hline $50-64$ & $221 \quad(937)$ & 307 & \\
\hline \multicolumn{4}{|l|}{ Reproductive history [n(\%)] } \\
\hline Previous induced abortion & $203 \quad(34)$ & $(32)$ & \multirow[t]{3}{*}{ NS* } \\
\hline Previous spontaneous abortion & $71 \quad(12)$ & (9) & \\
\hline Previous ectopic pregnancy & $(0.5)$ & $(0.8)$ & \\
\hline \multicolumn{4}{|l|}{ Deprivation category score $^{\dagger}[n(\%)]$} \\
\hline Affluent & $(15)$ & $(17)$ & \multirow[t]{3}{*}{ NS* } \\
\hline Intermediate & $(72)$ & (69) & \\
\hline Deprived & $(12)$ & (13) & \\
\hline
\end{tabular}

(62\%) vs $n=231(43 \%)$ for EMD and day case, respectively; $p<0.001$ ] and were more likely to be parous $(45 \%$ and $34 \%$ parous in EMD and day case, respectively, $p=0.003$ ) (Table 1 ).

\section{Unscheduled hospital re-attendances}

A total of $43 / 1128$ (4\%) women undergoing medical abortion made an unscheduled re-attendance (within 6 weeks) for a problem related to the abortion. There was no significant difference in the re-attendance rates between the EMD and day case groups, with 23 (4\%) and 20 (4\%) women making an unscheduled re-attendance after EMD and day case, respectively. However, re-attendance rates were significantly higher (in both treatment groups) among women who were $>56$ days' gestation, with 16/238 (6.7\%) women between 57 and 64 days' gestation making a unscheduled re-attendance compared to $27 / 847$ (3\%) women at $\leq 56$ days' gestation $(p=0.019)$.

All 43 re-attendances were due to heavy bleeding and/or pain. In 37 (86\%) of these unscheduled attendances an ultrasound was performed and bacteriological testing was performed in nine $(21 \%)$ cases. Three $(0.3 \%)$ women who had an abortion (at 60, 61 and 64 days' gestation) required a blood transfusion due to haemorrhage. In one of these cases, the haemorrhage occurred in a woman (who had planned to have EMD) on the same day that she received mifepristone. She expelled the pregnancy following mifepristone and never required misoprostol treatment. The other two cases of haemorrhage occurred in women treated as day cases, both of whom expelled the pregnancy during their day case treatment. However, the haemorrhage requiring blood transfusion actually occurred at 7 and 10 days after misoprostol treatment. One of these women also underwent a surgical evacuation of the uterus.

A further woman who was treated as a day case and who expelled the pregnancy at this time underwent a surgical evacuation of the uterus at 2 weeks after misoprostol treatment. This was conducted because an ultrasound scan to investigate persistent bleeding suggested features of a molar pregnancy. Subsequent pathology of uterine curettings was, however, normal. Another woman in the day case group who presented with pain 2 days after misoprostol underwent a laparoscopy to drain a simple ovarian cyst. All other unscheduled re-attendances were either managed expectantly or were given oral antibiotics for presumed infection (13 women were given oral antibiotics, $1 \%$ of all the women undergoing abortion).

There was no significant difference between groups in the timing of hospital re-attendances relative to the date of administration of mifepristone with a median interval of 19.5 [interquartile range (IQR) 5-25] days and 14.5 (IQR 8-25) days for EMD and day case groups, respectively.

A further 24 women [10 (2\%) and 14 (3\%) women in the EMD and day case groups, respectively] made unscheduled attendances at other departments within the hospital over the 6 weeks' post-abortion for a variety of medical complaints unrelated to the procedure.

Of the 590 women having EMD, 125 (21\%) failed to keep the scheduled routine follow-up to exclude ongoing pregnancy at 2 weeks. In the day case group, 208/533 (39\%) women did not pass products of 
Table 2 Method of contraception at discharge home provided to women undergoing medical abortion by either early medical discharge or day case procedure*

\begin{tabular}{|c|c|c|}
\hline Method & $\begin{array}{l}\text { EMD }(n=590) \\
{[n(\%)]}\end{array}$ & $\begin{array}{l}\text { Day case }(n=538) \\
{[n(\%)]}\end{array}$ \\
\hline Implant & $118(20)$ & $116(22)$ \\
\hline Injectable & $41 \quad(7)$ & $57(11)$ \\
\hline Combined oral contraceptive pill & $144(24)$ & $151(28)$ \\
\hline Progestogen-only pill & $59(10)$ & $31 \quad(6)$ \\
\hline Condoms & $34 \quad(6)$ & $25 \quad(5)$ \\
\hline None & $194(33)$ & $153(29)$ \\
\hline Fast-track appointment for intrauterine method ${ }^{\dagger}$ & $80(13)$ & $48 \quad(9)$ \\
\hline \multicolumn{3}{|c|}{$\begin{array}{l}\text { *There was no significant difference in the proportion of women in either group receiving effective hormonal } \\
\text { methods of contraception. Significantly more women in day case groups were fast-tracked to contraceptive services } \\
\text { for intrauterine methods ( } p=0.014) \text {. } \\
\text { 'Women were given an appointment at a local contraceptive service for intrauterine method insertion. } \\
\text { EMD, early medical discharge. }\end{array}$} \\
\hline
\end{tabular}

conception during their hospital admission and were given a follow-up appointment for 2 weeks later. Eighty-three $(40 \%)$ of these women failed to attend this scheduled follow-up appointment. Failure to keep a routine follow-up to exclude ongoing pregnancy was significantly less in the EMD group than in the day case group $(p<0.001)$. The proportion of women with an ongoing pregnancy after EMD or day case treatment was not significantly different, with 2/590 (0.3\%) women having EMD having an ongoing pregnancy after treatment compared to $2 / 538(0.3 \%)$ having day case treatment. The gestations at initial treatment in these ongoing pregnancies were 46 and 55 days for EMD, and 56 and 63 days for day case failures, respectively.

\section{Contraception at discharge}

There was no significant difference in the proportion of women who left with an effective hormonal (injectable, implant or pill) method of contraception, with $362(61 \%)$ in the EMD and $355(60 \%)$ women in the day case group receiving a hormonal method, respectively. The numbers of women in either group who were discharged home with a LARC method (progestogen-only injectable or implant method) was 159 $(27 \%)$ in the EMD and 173 (32\%) women in the day case groups, respectively. This difference in the proportion of women receiving LARC was of borderline significance $(p=0.055)$ (Table 2$)$. There was no significant difference in the proportion of women in either group receiving an implant with 118 (20\%) and 116 $(22 \%)$ women having an implant in the EMD and day case groups, respectively. There were, however, significantly more women in the EMD group who were given a 'fast-track' appointment arranged at the local family planning service for an intrauterine method to be fitted [ $n=80(13 \%)$ and $n=48(9 \%)$ of women in the EMD and day case groups, respectively, receiving a fast-track appointment for an intrauterine method; $p=0.014]$.

\section{Discussion}

This study showed that the unscheduled re-attendance rate at a hospital abortion service for a complication related to early medical abortion was low, and that there was no difference in unscheduled re-attendance rates between women choosing to go home soon after misoprostol (EMD) or women who are treated as a day case. Women who were over 8 weeks' gestation had higher unscheduled re-attendance rates in both treatment groups, but these rates did not differ significantly with treatment group; and even among women over 8 weeks' gestation, unscheduled re-attendance remained uncommon. Furthermore, the vast majority of re-attendances required expectant or medical management with oral antibiotic therapy. Indeed, the incidence of haemorrhage was no greater than that reported in the scientific literature. ${ }^{12}$ Furthermore, in none of the three cases of haemorrhage did this occur immediately following misoprostol treatment, which highlights how this complication would not have been prevented by hospital day case admission. Furthermore, the temporal distribution of unscheduled re-attendances in EMD and day case groups was comparable. This information is reassuring for the health professionals who may be considering establishing an EMD service within their abortion service, since it shows that allowing women to go home soon after misoprostol is safe and does not impact upon the emergency service any more than an existing day case medical abortion service already does.

Clearly, however, women in our study who chose to have an EMD were a self-selected group who may arguably be women who are better able to cope at home. Furthermore, women in the EMD group were more likely to be parous, a factor known to be associated with less reported pain, and were more likely to be at an earlier gestation, a factor known to be associated with less bleeding. ${ }^{13}$ Despite these differences in demographic characteristics between the groups, 
there was no significant difference in the proportion of women in each group from deprived postcode areas (depcat score). ${ }^{11}$ Although women in the EMD were on average 2 years older than their day case counterparts, the mean age of the women in both groups was nevertheless similar (mid-20s) and similar proportions of women in both groups had previously undergone an induced abortion.

Clearly, our study was unable to account for consultations to general practitioners as a result of postabortal morbidity. However, one might consider that unscheduled re-attendances to the hospital (as determined in this study) are likely to account for the more serious cases. In addition, the study design does not permit us to determine whether there was any difference between EMD and day case groups in terms of telephone calls made to the abortion service for a concern related to the procedure. Clearly, a prospective study that recorded all calls to the abortion service would be required to examine this scenario.

Although it has previously been shown in a large observational study of over 4000 cases of early medical abortion that addition of a further dose of misoprostol can reduce the ongoing pregnancy rate, we did not find a significant difference in the number of ongoing pregnancies following EMD treatment or day case treatment. ${ }^{14}$ While we do not know what proportion of women in the day case group received the extra dose of misoprostol, clearly it is reassuring that women in our study who chose EMD (and thus received a single dose of misoprostol only) were not disadvantaged by this. Further larger scale studies are, however, required.

Our study also showed that women choosing EMD were just as likely to leave hospital with an effective method of contraception as their day case counterparts. The NHS Quality Improvement Scotland standards for sexual health services recommend that at least $60 \%$ of women leave an abortion service with an effective method of contraception. ${ }^{15}$ Our study shows that this is achievable with both a day case and EMD medical abortion service. This is reassuring information for service providers, to know that women who choose EMD are not disadvantaged by the shortened hospital stay in terms of receiving effective contraception. Another reassuring finding was that similar proportions of women having EMD and day case treatment received implant contraception at discharge. It is to the credit of the nursing and medical staff working within the service that given the short stay of women in the EMD group that implant insertion could be organised for them before they left the premises. This does, however, highlight the importance of good contraceptive counselling before women attend for misoprostol administration.

Although immediate insertion of an intrauterine method has been shown to reduce the likelihood of a further abortion, ${ }^{16-18}$ it may not be feasible for all medical abortion services to provide this service, and clearly it is not possible to offer this to women choosing EMD, since they go home to expel the pregnancy. The Royal College of Obstetricians and Gynaecologists Service Standards for Gynaecology recommend that where immediate insertion is not possible 'fast-track' systems should exist between abortion services and specialist contraceptive providers for provision of intrauterine methods. ${ }^{19}$ In our study a higher proportion of women choosing EMD chose a 'fast-track' appointment than did their day case counterparts. While this may possibly reflect the differing characteristics of women in the two groups, this is also reassuring information for service providers and policymakers, since it shows that the shortened stay for EMD does not disadvantage women so far as receiving onward referral for specialist contraceptive fitting is concerned.

\section{Conclusions}

EMD is becoming an increasingly popular choice for women in early pregnancy who request a termination of pregnancy. Providing an EMD service alongside the more traditional day case medical abortion service does not appear to exert any additional pressure on an NHS hospital abortion service in terms of the proportions of women who make an unscheduled reattendance. Furthermore, the shorter stay of women choosing EMD does not have to disadvantage women in terms of receiving effective contraception on discharge from a well-organised abortion service.

\section{Competing interests None.}

Provenance and peer review Not commissioned; externally peer reviewed.

\section{References}

1 Fiala C, Winikoff B, Helström L, et al. Acceptability of home-use of misoprostol in medical abortion. Contraception 2004;70:387-392.

2 Clark WH, Hassoun D, Gemzell-Danielsson K, et al. Home use of two doses of misoprostol after mifepristone for medical abortion: a pilot study in Sweden and France. Eur J Contracept Reprod Health Care 2005;10:184-191.

3 Kopp Kallner H, Fiala C, Stephansson O, et al. Home selfadministration of vaginal misoprostol for medical abortion at 50-63 days compared with gestation of below 50 days. Hum Reprod 2010;25:1153-1157.

4 Schaff EA, Fielding SL, Westhoff C, et al. Vaginal misoprostol administered 1, 2, or 3 days after mifepristone for early medical abortion: a randomized trial. JAMA 2000;284:1948-1953.

5 Judiciary of England and Wales. BPAS-v-The Secretary of State for Health, 2011. http://www.judiciary.gov.uk/media/ judgments/2011/BPAS-secretary-state-for-health-judgment14022011 [accessed 18 May 2011].

6 Cameron S, Glasier A, Dewart H, et al. Women's experiences of the final stage of early medical abortion at home: results of a pilot survey. J Fam Plann Reprod Health Care 2010;36:213-216.

7 Lohr PA, Wade J, Riley L, et al. Women's opinions on the home management of early medical abortion in the UK. J Fam Plann Reprod Health Care 2010;36:21-25.

8 Tupper C, Speed Andrews S. Setting up an outpatient service for early medical termination. J Fam Plann Reprod Health Care 2007;33:199-202. 


\section{Astle et al.}

9 Department of Health. Evaluation of Early Medical Abortion (EMA) Pilot Sites, 2008. http://www.dh.gov.uk/en/ AdvanceSearchResult/index.htm? searchTerms =early + medical + abortion [accessed 9 May 2011].

10 NHS National Services Scotland. Scottish Abortion Statistics Information Services Division, 2011. 2011. http://www. isdscotland.org/isd/1918.html\#Activity_in_2009 [accessed 9 May 2011].

11 McLoone P. Carstairs Scores for Scottish Postcode Sectors from the 2001 Census - Report, 2000. 2000. http://www. sphsu.mrc.ac.uk/sitepage.php? page $=$ carstairs [accessed 9 May 2011].

12 Grimes DA. Medical abortion in early pregnancy: a review of the evidence. Obstet Gynecol 1997;89:790-796.

13 Bartley J, Tong S, Everington D, et al. Parity is a major determinant of success rate in medical abortion: a retrospective analysis of 3161 consecutive cases of early medical abortion treated with reduced doses of mifepristone and vaginal gemeprost. Contraception 2000;62:297-303.
14 Ashok PW, Templeton A, Wagaarachchi PT, et al. Factors affecting the outcome of early medical abortion: a review of 4132 consecutive cases. BJOG 2002;109:1281-1289.

15 Healthcare Improvement Scotland. Standards for Sexual Health Services. 2008. http://www.healthcareimprovementscotland. org/programmes/reproductive_maternal_child/programme resources/sexual_health_standards.aspx [accessed 9 May 2011].

16 Heikinheimo O, Gissler M, Suhonen S. Age, parity, history of abortion and contraceptive choices affect the risk of repeat abortion. Contraception 2008;78:149-154.

17 Roberts H, Silva M, Xu S. Post abortion contraception and its effect on repeat abortions in Auckland, New Zealand. Contraception 2010;82:260-265.

18 Goodman S, Hendlish SK, Reeves MF, et al. Impact of immediate postabortal insertion of intrauterine contraception on repeat abortion. Contraception 2008;78:143-148.

19 Royal College of Obstetricians and Gynaecologists. Standards for Gynaecology - Report of a Working Party. 2008. http:// www.rcog.org.uk/catalog/book/standards-gynaecology-reportworking-party [accessed 9 May 2011]. 